\title{
Histopathological features of subcutaneous mycosis: a retrospective study
}

\author{
Subhashini R. ${ }^{1}$, Bhat R.V. ${ }^{2}$ \\ ${ }^{1}$ Dr. Subhashini R., Postgraduate, ${ }^{2}$ Dr. Ramachandra V Bhat, Professor \& Head, Both authors are affiliated with \\ Department of Pathology, Indira Gandhi Medical College and Research Institute, Puducherry, India. \\ Corresponding Author: Dr. Ramachandra V Bhat, HOD \& Professor, Department of Pathology, Indira Gandhi Medical \\ College and Research Institute, Puducherry. E-mail id: rvbhatpath@gmail.com
}

\begin{abstract}
Background: Subcutaneous fungal infection is commonly seen in tropical and subtropical countries particularly in India. The lesions are usually presented as swelling, in the localized form. Subcutaneous mycosis is caused by penetration of causative fungi into the subcutaneous tissue. Histopathology is one of the main tools of diagnosis in mycology as it has the advantages of early diagnosis compared to culture, low cost and it provides a presumptive identification of the infecting fungus. It also gives information about tissue reaction and supplement culture report. It gives the idea about the fungus isolated in culture is contaminant or pathogenic. Many a times tissue might be sent in formalin as fungal infection is not suspected clinically. Role of histopathologist is very important in diagnosing these subcutaneous fungal lesions. Material \& Methods: A retrospective study was conducted on subcutaneous fungal infections seen between January 2014 to December 2018 in the Department of pathology, IGMC\&RI, Puducherry. Twenty-two patients with biopsy proven subcutaneous fungal infections were included in the study. In the present study, the varied histopathological features like type of inflammatory response, presence of granulomas, necrosis, eosinophils and abscess formation were seen in the tissue sections. Results: The most common histopathology feature was giant cell reaction seen in 21 (95.5\%) cases, followed by epithelioid cell granuloma in 13(59\%) cases and areas of necrosis in 13 (59\%) cases. Eight (36\%) cases showed numerous eosinophilic infiltration 10(46\%) cases of scant eosinophils and 4 (18\%) cases showed absent eosinophils. These fungal structures were identified in H\&E stain itself in 13 (59\%) cases. Conclusion: Histopathological study is most important in subcutaneous mycosis as most of the time diagnosis is unsuspected and delayed because of its rarity and varied presentation. Fungal infection should be suspected in cases with localized cystic swelling. High index of suspicion, careful microscopic examination and special stains are important for accurate diagnosis.
\end{abstract}

Keywords: Fungal, Subcutaneous, Mycosis, Histopathology

\section{Introduction}

Subcutaneous fungal infection is commonly seen in tropical and subtropical countries particularly in India. The lesions are usually presented as swelling, in the localized form [1]. These infections are more common in the adult males from the rural areas who are predisposed to trauma and contact with soil and decaying vegetation [2]. As it is presented with localized swelling without signs of inflammation it was clinically mistaken for other benign lesions like lipoma, hemangioma, abscess etc [3]. Although culture is considered as gold standard for etiological diagnosis

Manuscript received: $4^{\text {th }}$ October 2019

Reviewed: $14^{\text {th }}$ October 2019

Author Corrected: $20^{\text {th }}$ October 2019

Accepted for Publication: 24 ${ }^{\text {th }}$ October 2019 fungal infections, it may not be useful in such cases as clinically fungal infection is not suspected and not sent for culture. Disadvantage of Culture is it is a slow method compared to other methods and some of these fungi may not grow in culture. Histopathology examination is the main investigation required for diagnosis of subcutaneous fungal infections.

It is one of the methods for diagnosing fungal infections along with other methods like specific antigen detection, antibody detection, specific metabolite detection and recent PCR technique [4]. All other nonculture methods except histologic method cannot detect all the types of fungi and are not available 
widely. Histopathologic examination can also provide insight into the diagnostic significance of some culture isolates. Histopathological evaluation of granulomatous inflammation, giant cell reaction, necrotizing inflammation etc must include careful search for fungal elements. The presence of fungus in the tissue sections provides an indisputable evidence of invasive infection.

Because of their size, presence of pigments and morphologic diversity, many fungi can be seen in tissue sections by conventional light microscopic examination of Hematoxylin and Eosin (H\&E) stained sections in the tissues.

However, special stains are essential to rule out artifacts and when fungi are sparse.

Gomori Methenamine Silver (GMS) and Periodic acidSchiff (PAS) are the two most common stains used to look for fungi in tissues and in cytology specimens in the daily practice of pathology.

Fungi usually occur either as hyphae, budding yeast, endosporulating spherules, or a combination of these forms. Tissue reactions may vary depending on the type of fungi, its virulence, duration and host immune response [5]. The objective was to study the various histopathological features which are associated with subcutaneous mycosis.

\section{Materials and Methods}

Study settings and duration: It is a retrospective study conducted on cases of subcutaneous fungal infections seen between 2014 to 2018 in the Department of Pathology, Indira Gandhi Medical College and Research Institute, Puducherry.

Type of study: Retrospective Observational study

\section{Original Research Article}

Inclusion criteria:

All cases reported as Subcutaneous mycosis by histopathology in Department of Pathology during the study period from January 2014 to December 2018.

\section{Exclusion criteria:}

Duplication of data from cases with repeat biopsy. Fungal infections reported as Superficial and Deep mycoses

Ethical consideration \& permission: This study was carried out after obtaining due permission from Institute Ethics Committee (IEC No.4/174/IEC/PP/2019). As it is a retrospective and lab data based in nature, no ethical issues involved.

Sampling methods: For this study, all cases were reviewed with biopsy proven subcutaneous mycosis during the study period. Total of 22 cases were included in the study.

Data collection procedure: Patients clinical data was collected from Medical Records Department (MRD) and it was analyzed including patients age, gender, site, duration, multiplicity, predisposing factors like history of trauma (thorn prick) and co-morbid conditions. The histopathological features were studied from all cases of subcutaneous mycosis reported during the study period, by retrieving the H\&E slides and fungal morphology studied using special stains such as Periodic acid Schiff (PAS) and Gomori Methanamine Silver (GMS). Final diagnosis was based on identification of fungal structures in H\&E and / or in special stains.

Data analysis: Data entry was done using MS excel sheet and analyzed using SPSS software (version 20). Categorical variables was expressed using frequency and percentages. Quantitative variables were expressed using mean. The demographic data like age, sex etc are expressed in percentage and ratio.

\section{Results}

Clinical characteristics: A total of 22 patients were evaluated for subcutaneous fungal infections. Out of which $12(54.5 \%)$ were male and $10(45.5 \%)$ were female patients and the male: female ratio was 1.2:1. The age group varied from 5-80 years. 15 (68\%) patients were aged between 20-60 years, $6(27.5 \%)$ were $>60$ years and $1(4.5 \%)$ patient was $<20$ years of age. The most common site of involvement was the lower limb seen in $12(54.5 \%)$ cases, followed by upper limb in $8(36.5 \%)$ cases, back in $1(4.5 \%)$ case and abdominal wall in $1(4.5 \%)$ cases. The history of trauma was obtained in only one $(4.5 \%)$ case. There were no other comorbid conditions like diabetes mellitus, cancer, HIV etc in any of these cases. None of the cases showed multiple lesions or systemic manifestations. The most common clinical diagnosis of subcutaneous mycosis was sebaceous cyst in $12(54.5 \%)$ cases, followed by lipoma in $3(14 \%)$ cases and fungal abscess in $3(14 \%)$ cases and ganglion in $2(9 \%)$ cases, soft tissue tumor and retrocalcaneal bursal cystin one case each.

Gross features: The most common type of specimen received was cyst with thick fibrous wall in $11(50 \%)$ cases, followed by grayish brown nodule in 7 (32\%) cases and as fragmented bits in $4(18 \%)$ cases.

Pathology Update: Tropical Journal of Pathology \& Microbiology Available online at: www.medresearch.in 802 | P a g e 


\section{Original Research Article}

Histopathology features: The various histopathological features of subcutaneous mycosis are studied (Table 1). Of which the most common histopathological feature was foreign body giant cell reaction seen in $21(95.5 \%)$, followed by well-formed epithelioid cell granuloma 13 (59\%) cases and areas of necrosis in 13(59\%) cases (Figure 1). Eight (36\%) cases showed numerous eosinophilic infiltration and 10(46\%) cases showed scant eosinophils. The fungal elements were most commonly seen in cyst wall in $15(68 \%)$, followed by within the multinucleated giant cells in $7(32 \%)$ cases, necrotic debris in $6(27 \%)$ and in one case $(4.5 \%)$ seen in granuloma. The most common fungal structure identified in this study was pigmented, branching, septate hyphae in $15(68 \%)$, followed by non- pigmented septate acute angle branching hyphae in $3(13.6 \%)$ cases.

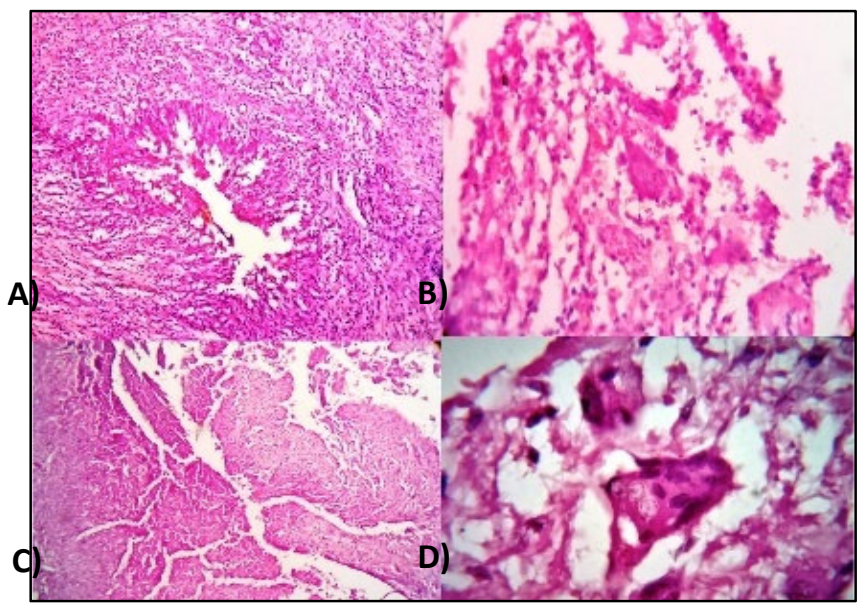

Figure1: (A) H\&E 40X shows cyst wall; (B) H\&E 40X shows inflammatory infiltrate with multinucleate giant cells (C) H\&E 40X shows necrotic debris; (D) H\&E 100X shows foreign body multinucleate giant cells

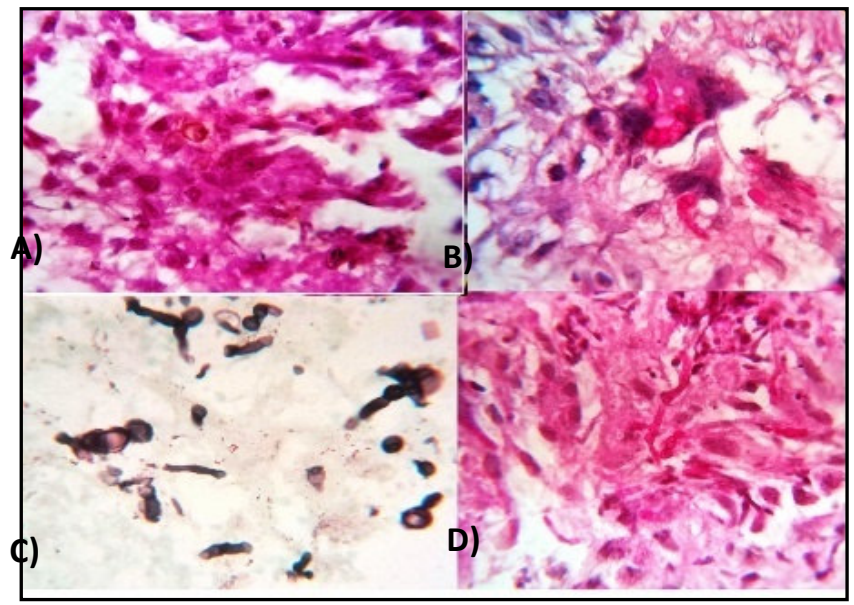

Figure 2: (A) H\&E 100X shows pigmented branching fungal hyphae; (B) PAS 100x shows fungal hyphae within the giant cells; (C) GMS 100x shows branching septate fungal hyphae; (D) PAS 100x shows septate hyphae

These fungal structures were identified in H\&E stain itself in 13 (59\%) cases on careful examination .Special stain like Periodic Acid Schiff (PAS) and Gomorimethanamine silver (GMS) stain showed pigmented septate branching fungal hyphae in $15(68 \%)$ cases, non pigmented septate branching hyphae in $3(13.6 \%)$ cases (Figure 2)and $3(13.6 \%)$ cases showed fungal spores with hyphae and one (4.5\%) case of medlar bodies. Among 22 cases totally, $15(68 \%)$ cases were reported as phaeohyphomycosis followed by $6(27 \%)$ cases of hyalohypomycosis and one case of chromoblastomycosis $(4.5 \%)$.

In three cases which were not included in this study, tissue histopathological features were very much suggestive of subcutaneous mycosis, but special stains like PAS \& GMS were failed to demonstrate fungal organisms and were reported as suspicious of fungal infection. It was advised for repeat biopsy and microbiological examination. 
Original Research Article

Table-1: Various histopathological features seen in subcutaneous mycosis

\begin{tabular}{|l|c|}
\hline Histopathology Features & No of Cases (\%) \\
\hline 1) Epithelioid granuloma & $13(59 \%)$ \\
\hline 2) Giant cell reaction & $21(95.5 \%)$ \\
\hline 3) Eosinophilic infiltration & $8(36 \%)$ \\
\hline a) Numerous & $10(45.5 \%)$ \\
\hline b) Scant & $13(59 \%)$ \\
\hline 4) Necrosis & $16(72 \%)$ \\
\hline 5) Cystic change & $4(18 \%)$ \\
\hline 6) Neutrophilic abscess & \\
\hline
\end{tabular}

\section{Discussion}

The tissue harboring the fungal infection may be of different types viz., skin, subcutaneous tissue and deeper organs like lung, central nervous system, bone, liver etc., and accordingly the mycoses are divided into different types. Subcutaneous fungal infections or mycoses include fungal infections involving the lower dermis and the subcutaneous tissue. They usually occur following invasion of the fungi into the subcutaneous layer by accidental traumatic penetration of thorns or wooden stick. The various other manifestations of subcutaneous fungal infections in literature are cyst, plaque, ulcer, scaly lesion, verrucous growth, warty plaque, abscess and keratotic macerated lesions [1]. In present study, the most common manifestation was cystic lesion. The common site of presentation is upper and lower limbs over fingers, wrist, knee or ankles and less commonly on the scalp, neck and face $[2,6]$.

Hematoxylin \& Eosin is a versatile stain that enables the pathologist to evaluate the host response, including the chronic inflammation, granulomas, eosinophilic infiltration, abscess formation and Splendore-Hoeppli phenomenon, and to detect other micro-organisms. It is the stain of choice to confirm the presence of naturally pigmented fungi, and to demonstrate the nuclei of yeast-like cells. However, there are drawbacks to using just the H\&E stain for fungal diagnosis. It is often difficult to distinguish poorly stained fungi from tissue components, even at higher magnifications. When sparse, fungi are easily overlooked in H\&E stained sections. The morphologic features may not be evident and sometimes may be misleading. Most fungi can be readily demonstrated with the common special stains, Gomori's methenamine silver (GMS) and periodic acid-Schiff (PAS) [7].

Morphologically fungi may grow as yeast forms, hyphae (molds) and dimorphic forms. In yeast forms, the individual fungi are seen either in short chains or are individually separated. They are round to oval and reproduce by budding. In candida species, the buds often do not detach from each other and thus form a chain of elongated yeast cells which are known as pseudohyphae. Hyphae or molds are multicellular thread like filaments which can grow and divide at the tips. Hyphae may produce conidia which can disseminate through air. Dimorphic fungi can be present in either forms. They show yeast form in human body temperature and mold forms in room temperature. Morphological parameters which may help to differentiate different types of fungi on histology include estimation of size, the predominant form of the fungus (yeast, hyphae or pseudohyphae), presence or absence of capsule, thickness of the capsule, budding (single or multiple, narrow or wide based), presence or absence of septae and branching pattern in hyphae etc [8].

The common subcutaneous mycoses are phaeohyphomycosis, hyalohyphomycosis, mycetoma, chromoblastomycosis, zygomycosis, sporotrichosis, and rhinosporidiosis [9]. In the present study phaeohyphomycosis was the most common type of subcutaneous mycosis. Phaeohyphomycosis is caused by brown pigmented fungi having melanin in their cell walls. Melanin acts as a virulence factor because of its scavenging effects over free radicals and hypochlorite produced by phagocytic cells, and moreover it binds to hydrolytic enzymes. This may be the explanation for infection in immunocompetent hosts [10]. The most common etiological agents of subcutaneous phaeohyphomycosis are Exophialajeanselmi followed by Exophialadermatitidis [6]. The genus Exophialais widely distributed in the environment and may cause infections in both immunocompromised (human immunodeficiency virus patients, transplant recipients, debilitating chronic diseases, diabetes, and immunosuppressive therapy) and rarely, in immunocompetent persons. E. jeanselmius usually causes mild cutaneous and subcutaneous infections which are often localized and solitary (phaeohypomycotic cyst) [11]. Other fungal species causing this unique infection include Phialophora, Cladosporium, Wangiella, Fonsacaea, Alternaria, Bipolaris, and Curvularia species [12]. Histopathology of phaeohyphomycosis shows 
Original Research Article

inflammatory cells with multinucleated giant cells and epithelioid cells along with the presence of brown pigmented septate hyphae with acute angle branching [13]. The second most common subcutaneous mycosis encountered in this study was hyalohypomycosis. Hyalohyphomycosis is characterized by the presence of hyaline hyphae without the presence of pigment in the wall. It is a broad group which includes the following species namely Fusarium, Penicillium, Scedosporium, Acremonium, Paecilomyces, Aspergillus etc [1]. In the study by Bhat et al [14] the mean age of patients with subcutaneous infection was 49.4 years and the youngest age was 14 years and was more prevalent in rural areas. The most common site affected was lower extremities (64\%). The present study also shown similar findings.

In the present study, phaehypomycosis was the most common type encountered. In a study by Abraham et al., they have described seven cases of subcutaneous phaeohyphomycosis from India [15]. In another study by O'Donnell et al described nine cases of patients with subcutaneous phaehypomycosis [9]. In a study done by Sivayogana R et al. the most common subcutaneous infection was mycetoma ( $43 \%$ ) followed by phaeohypomycosis (32\%) [2]. Changes in the tissue as a reaction to the infection may include allergic reaction, acute and / or chronic reaction, granuloma formation, necrosis and vascular invasion [8].

Table-2: Histopathological features of subcutaneous mycosis

\begin{tabular}{|l|c|c|c|}
\hline \multicolumn{1}{|c|}{ Histopathology Features } & Gayathri P et al [1] (2015) & $\begin{array}{c}\text { Verma et al [3] } \\
\text { (2018) }\end{array}$ & Present study \\
\hline $\begin{array}{l}\text { Foreign body } \\
\text { multinucleated giant cells }\end{array}$ & $100 \%$ & $66 \%$ & $95.5 \%$ \\
\hline Epithelioid granuloma & $100 \%$ & $66 \%$ & $59 \%$ \\
\hline Cyst wall with inflammation & $100 \%$ & $14.3 \%$ & $72 \%$ \\
\hline Inflammatory reaction & $\begin{array}{c}\text { Acute and chronic } \\
\text { inflammatory infiltrate }\end{array}$ & $\begin{array}{c}\text { Eosinophilic } \\
\text { Infiltration } 81 \%\end{array}$ & $\begin{array}{c}\text { Eosinophilic } \\
\text { infiltration } 81.5 \%\end{array}$ \\
\hline Fungal elements in H\&E & $\begin{array}{c}100 \% \\
\text { (only pheohyphomycosis) }\end{array}$ & - & $59 \%$ \\
\hline
\end{tabular}

Common biopsy findings in these infections were giant cell reaction, granuloma formation, cystic change and eosinophilic infiltration. Most common histopathological finding encountered was multinucleated giant cell formation. Similar observation was made by Gayathri P et al [1] and Verma et al [3] This is expected as macrophages try to engulf the fungal elements resulting in giant cell formation. Granulomatous inflammation was seen in more than half of the cases which stressed the need for careful search with deeper sections and special stains whenever there is a granulomatous inflammation in subcutaneous tissue. Another important finding was formation of cystic lesion with fibrous wall particularly in phaehyphomycosis as observed in study conducted by Gayathri P et al [1]. Heavy eosinophil infiltration is seen in only 6 cases (36\%).

The fungal elements were most commonly seen in cyst wall in $15(68 \%)$, followed by within the multinucleated giant cells in 7 (32\%) cases. These are the key areas one has to search for fungal elements in suspected subcutaneous mycosis, particularly when patient is immunocompetent. The present study reiterates the fact that no single histological feature can be taken as most important diagnostic clue in subcutaneous mycosis and the histopathological features may vary depending on the nature of organism, virulence, host factors and the immunity [16].

Limitations: The limitations of the present study were number of cases were less and the microbiological culture study and species identification was not done.

\section{Conclusion}

Histopathological study is most important in subcutaneous mycosis as most of the time diagnosis is unsuspected clinically and delayed because of its rarity and varied presentation. Fungal infection should be suspected in cases with localized cystic swelling. High index of suspicion, careful microscopic examination and special stains are important for accurate diagnosis.

\section{What the study adds to the existing knowledge?}

The study emphasized the role of histopathological examination and special stains in diagnosing clinically unsuspected cases of subcutaneous mycosis. Study findings may help the histopathologists to find areas in tissue biopsies where to look for the fungal elements. 


\section{Original Research Article}

\section{Author's contributions}

Dr. Subhashini R: Collected the data, done HPE slide analysis, data statistical analysis and article writing part

Dr. Ramachandra V Bhat: Conception of study, getting necessary permissions, contributed to data analysis, approved the submitted version.

Funding: Nil; Conflict of Interest: None initiated Permission from IRB: Yes

\section{References}

1. Priyadharshini G, Varghese RG, Phansalkar M, Ramdas A, Authy K, Thangiah et al. Subcutaneous Fungal Cysts Masquerading as Benign Lesions- A Series of Eight Cases. J Clin and Diagn Res. 2015;9 (10): 1-4. doi: 10.7860/JCDR/2015/14157.6637.

2. Sivayogana R, Madhu R, Ramesh A, Dhanalakshmi UR. A Prospective Clinico mycological study of deep mycoses in a tertiary centre in Tamil Nadu. Int $\mathbf{J}$ Res Dermatol. 2018; 4(2):126-135. doi: http://dx.doi.org/10. 18203/issn.2455-4529.IntJResDermatol20181482.

3. Verma S, Thakur BK, Raphael V, Thappa DM. Epidemiology of subcutaneous mycoses in northeast India: A retrospective study. Indian J Dermatol 2018; 63 (6): 496-501.doi: 10.4103/ijd.IJD_16_18.

4. Rickerts V, Mousset S, Lambrecht E, Tintelnot K, Schwerdtfeger R, Presterl E et al. Comparison of histopathological analysis, culture, and polymerase chain reaction assays to detect invasive mold infections from biopsy specimens. Clinical Infectious Dis. 2007; 44 (8):1078-1083. doi.org/10.1086/512812.

5. Schwartz J. The diagnosis of deep mycoses by morphologic methods. Hum Pathol. 1982;13(6):519533. doi: 10.1016/s0046-8177(82)80267-0.

6.Chintagunta S, Arakkal G, Damarla SV, Vodapalli AK. Subcutaneous phaeohyphomycosis in an immunocompetent individual: a case report. Indian Dermatol OnlineJ.2017;8(1):29-31.doi:10.4103/2229-

5178.198770
7. Chandler FW, Watts JC. Fungal Diseases. In: Damjanov I, Linder J, editors. Anderson's Pathology. $10^{\text {th }}$ ed. St. Louis: Mosby; 1996.pp 951-962.

8. Guarner J, Brandt ME. Histopathologic diagnosis of fungal infections in the 21st century. Clinic Microbiol Rev. 2011;24(2):247-80. doi: 10.1128/CMR.00053-10.

9. O'Donnell PJ, Hutt MS. Subcutaneous phaeohyphomycosis: a histopathological study of nine cases from Malawi. J Clin Path. 1985;38(3):288-292. doi: http:// dx.doi.org/10.1136/jcp.38.3.288.

10. Mishra D, Singal M, Rodha MS, Subramanian A. Subcutaneous phaeohyphomycosis of foot in an immunocompetent host. J Lab Physicians. 2011;3(2): 122-124. doi: 10.4103/0974-2727.86848.

11. Madke B, Khopkar U. Pheohyphomycotic cyst. Indian Dermatol Online J. 2015;6(3):223-225. doi: 10. 4103/2229-5178.156429.

12. Revankar SG. Phaeohypomycosis. Infect DIS Clin North Am. 2006; 20(3):609-20. doi:10.1016/j.idc. 2006. 06.004

13. Sharma NL, Mahajan V, Sharma RC, Sharma A. Subcutaneous pheohyphomycosis in India - a case report and review. Int J Dermatol. 2002;41(1):16-20. https://doi.org/10.1046/j.1365-4362.2002.01337.

14. Bhat RM, Mo nteiro RC, Bala N, Dandakeri S, Martis J, Kamath GH et al. Subcutaneous mycoses in coastal Karnataka in south India. Int J Dermatol. 2016; 55(1):70-78. doi: 10.1111/ijd.12943.

15. Abraham LK, Joseph E, Thomas S, Matthai A. Subcutaneous phaeohyphomycosis: a clinicopathological study. Int Surg J. 2014; 1(3):140-143. doi: 10. 5455/2349-2902.isj20141106.

16. Pang KR, Jashin WJ, Huang DB, Tyring SK. Subcutaneous fungal infections. Dermat Ther. 2004; 17(6): 523-31. doi:10.1111/j.1396-0296.2004.04056.x.

\section{How to cite this article?}

Subhashini R, Bhat R.V. Histopathological features of subcutaneous mycosis: a retrospective study. Trop J Path Micro 2019;5(10):801-806.doi:10.17511/jopm.2019.i10.08. 